\title{
La coalescence dans l'industrie
}

\author{
Coalescence in industry
}

\author{
J.-M. Fitremann
}

Société Hydroscience, Nantes

La coalescence est un phénomène qui peut se produire dans les écoulements de fluides lorsque deux phases ou plus sont en présence. Pour certains, la coalescence évoque la fusion de deux globules en un seul au sein d'un fluide, pour d'autres une fusion de globules sur un support adéquat par impact et ruissellement. Le deuxième cas fait intervenir trois phases dont un solide et donc trois tensions interfaciales, contrairement au premier qui n'en fait intervenir qu'une seule. Nous avons ces deux types de phénomènes présents à l'esprit dans nos travaux de réflexion, bien que la plupart des exemples soient du premier type.

\section{Coalescence en milieu gaz-liquide et en milieu liquide-liquide}

Selon le rapport des densités de la phase dispersée à la phase continue, on a trois cas :

$\rho_{d} \ll \rho_{d} \quad$ bulles dans un liquide

$\rho_{d} \sim \rho_{c} \quad$ gouttes dans un autre liquide

$\rho_{d}>\rho_{c} \quad$ gouttes dans un gaz

Le troisième cas est rarement préoccupant car l'inertie importante de la phase dispersée est telle que tout choc conduit à une fusion immédiate des gouttes. Néanmoins, le problème des fréquences de collisions est général et s'applique aux trois cas.

Dans les deux premiers cas, lors de l'approche de deux globules, il se forme un film intersticiel de liquide qui se draine sous l'action des forces de pression. La variété des circonstances où se produit la coalescence entraîne celle des forces mises en jeu. On peut distinguer les forces motrices du drainage, et les forces retardatrices.

\section{Forces motrices du draînage}

La formation du film intersticiel peut se produire de différentes façons, soit :

\section{Collisions : Forces d'inertie}

- des globules (faible dans le cas des bulles)

- du liquide environnant

Dans le cas d'une collision, les forces de drainage sont essentiellement des forces d'inertie, instationnaires, dynamiquement équilibrées par la tension interfaciale.

Les collisions peuvent provenir d'un mouvement défini, avec écoulement laminaire, ou d'un mouvement turbulent, de caractère aléatoire.

Les phénomènes de choc et de rebond sont mal connus. Le lien entre les caractères des collisions (fréquences, vitesses d'impact, etc.) et ceux du mouvement laminaire (cisaillement) ou turbulent (énergie, échelles, dissipation) est également un problème à résoudre.

\section{Empilement : Forces de pesanteur}

Un globule se maintient sous ou sur une interface globalement horizontale tant que le film intersticiel n'est pas rompu. Les forces sont cette fois statiques (quasiment). Dans certaines circonstances, il y a un grand nombre de globules empilés sous forme d'un lit compact. Il y a des coalescences globule-globule et globule-interface.

\section{Autre: Forces élastiques}

On peut artificiellement presser deux globules l'un contre l'autre, la déformation élastique étant équilibrée par les forces de tension. Cette situation peut aussi se produire dans certains dispositifs.

\section{Forces retardatrices du draînage}

Le drainage du film n'est pas immédiat. On compte un nombre important de mécanismes ralentissants : 


\section{Viscosité volumique}

La viscosité des deux phases joue un rôle retardateur du mouvement des fluides, et surtout du film mince formé entre les globules.

\section{Viscosité surfacique}

La viscosité interfaciale s'oppose également au mouvements rapide de l'interface.

\section{Effet}

Le gradient de tension interfaciale entraîne une discontinuité des contraintes tangentielles et donc une modification des mouvements des fluides par rapport au cas d'une tension constante.

Les variations de tension interfaciales sont le plus souvent liées à celle d'un constituant absorbé sur les interfaces et/ou partiellement en solution.

\section{Forces moléculaires}

Il existe le plus souvent des forces électriques à courte portée de nature :

- moléculaire - ces forces jouent un rôle dans les mécanismes de mise en contact des globules, d'empilement et d'agrégation, de flocculation. Elles jouent aussi dans le draînage du film en s'ajoutant aux forces de pression.

\section{Rupture des films intersticiels}

L'amincissement du film sous l'action des forces de draînage conduit à la rupture lorsque l'épaisseur tombe au-dessous d'une épaisseur critique au-dessous de laquelle la cohésion moléculaire du liquide est insuffisante. Les épaisseurs de rupture observées sont en général, très supérieures à celles que prédisent les théories moléculaires sans qu'on en ait d'explication satisfaisante.

\section{Stabilité des films}

Dans certains cas, les forces de répulsion moléculaires sont suffisantes pour équilibrer les forces de pression. Les films intersticiels sont alors stables et la coalescence ne se produit plus. On doit distinguer ce phénomène d'un draînage excessivement lent, par exemple, lorsque les interfaces sont " rigidifiés " par l'adsorption de surfactant et que l'écoulement dans le film est très visqueux et les forces de draînage faibles.

Il existe un autre cas de stabilité qui est connu sous le nom de micro-émulsion et exposé séparément.

\section{Distinction entre phénomènes gaz-liquide et liquide- liquide}

Dans le cas de mélanges gaz-liquide ou vapeur-liquide, le draînage est en général rapide sauf si les interfaces sont " rigidifiés " par la présence de surfactants comme dans le cas des mousses. La viscosité interfaciale est en général très faible, à la limite du mesurable.

Les mélanges gaz-liquide sont caractérisés également par un glissement notable des bulles de gaz. Ce glissement est une source supplémentaire de turbulence dans le cas de bulles assez grosses (par exemple supérieures à $0,5 \mathrm{~mm}$

de diamètre pour l'air et l'eau), donc d'augmentation des fréquences de collision.

A l'inverse, la turbulence peut également provoquer la rupture des bulles et en limiter le diamètre. Il y a donc fréquemment compétition entre rupture et coalescence pour établir le diamètre moyen des bulles.

Dans le cas d'un mélange liquide-liquide, la plus faible différence de densité limite les vitesses de glissement. L'inertie plus grande rend l'échelle de temps des phénomènes sensiblement plus grande également.

Une possibilité nouvelle offerte par la deuxième phase liquide est de pouvoir dissoudre des molécules insolubles dans la première phase. Lorsque ces molécules s'adsorbent sur l'interface, on peut arriver à une distribution interfaciale de surfactants et co-surfactants qui peut modifier de façon considérable ses propriétés physiques. Les modifications peuvent inhiber le draînage des films en le ralentissant ou en l'arrêtant totalement. C'est ainsi que l'on peut stabiliser des émulsions par addition d'un couple de surfactants.

Un autre effet possible est l'abaissement de la tension interfaciale, couramment utilisé pour faciliter l'émulsification par des moyens mécaniques.

L'abaissement de la tension interfaciale dépend de la concentration surfacique des surfactants, qui elle-même dépend de l'aire interfaciale volumique. Dans certains cas, l'augmentation de l'aire interfaciale par fractionnement de la phase dispersée est telle que la tension interfaciale peut devenir nulle ou même négative. Dans ce cas, le diamètre des gouttes se stabilise à une valeur très petite qui donne une tension interfaciale tout juste positive. On appelle ce type de mélange thermodynamiquement stable microémulsion. Il se comporte alors comme une phase ordinaire, distincte de l'une ou l'autre des phases originales. Il peut également y avoir deux micro-émulsions stables, de dispersion opposée, issues de deux phases originales identiques.

\section{Exemples de situations industrielles} où la coalescence joue un rôle

La coalescence peut être recherchée, activée ou nuisible et partiellement ou totalement inhibée. Dans certains processus, on peut vouloir l'inhiber un certain temps, la promouvoir ensuite ou l'inverse. Dans bien des cas, le contrôle de ce phénomène est imparfait. Nous essayons de décrire les processus les plus fréquemment rencontrés.

\section{Ecoulements de mélanges}

L'écoulement d'un mélange gaz-liquide, vapeur-liquide ou liquide-liquide dans une conduite produit, loin de l'injection où se fait le mélange, une configuration stable qu'on décrit traditionnellement comme :

- à bulles, à gouttes

- à poches, à bouchons si l'une des phases est dispersée finement si des volumes de plusieurs diamètres de conduite sont générés 
- annulaire

si les deux phases sont concentriques

- séparée (ou "stratifiée") si les deux phases sont superposées

Les transitions entre ces configurations par variation des débits provient soit d'instabilité dynamiques, soit de la coalescence de bulles ou de gouttes qui ne sont pas compensées par les mécanismes de fractionnement. L'établissement de la configuration à poches/bouchons en particulier est lié directement au rythme du processus de coalescence.

Ces écoulements se rencontrent dans de nombreuses branches de l'industrie, à savoir :

- transport de produits pétroliers par pipe-lines, sea-lines etc.;

- extraction des hydrocarbures;

- génie nucléaire; réacteurs bouillants, sécurité du réacteur à eau pressurisée, à sodium.

Dans ces différents cas, des modèles numériques sont utilisés pour prédire les pertes de pression, aire interfaciale volumique etc. pour chaque configuration. La prévision des domaines paramétriques des diverses configurations est très importante et peut être mieux appréciée par la connaissance des lois de coalescence. Par ailleurs, on injecte parfois l'une des phases sous forme d'une configuration qui se transforme ultérieurement: injection de bulles donnant finalement des poches, par exemple. La connaissance des fréquences de coalescence permettrait d'évaluer a priori les longueurs d'établissement d'une configuration, grandeur d'intérêt notable.

\section{Séparation}

Le " moteur" de la séparation de phases est gravitaire, centrifuge, parfois autre (électrique, gradient de pression). $\mathrm{Ce}$ " moteur" produit d'abord une sédimentation. Les globules se tassent ensuite sous ou sur l'interface séparant le mélange de la phase libérée si le temps de coalescence est long. Sinon, chaque globule coalesce individuellement avec l'interface.

Ainsi, il se forme fréquemment un lit dense de globules au sein duquel se produisent continuellement des coalescences. A l'interface lit-phase libérée, d'autres coalescences ont lieu, ce sont ces dernières qui libèrent la phase dispersée.

En connaissant mieux les lois de coalescence en lit dense, il serait possible de prévoir leur épaisseur et donc d'améliorer les performances des séparateurs ou de mieux déterminer leur géométrie.

On trouve des phénomènes similaires dans les coalescences électrostatiques, bassins de sédimentation, etc.

Dans de nombreux cas, on aide la séparation par des phénomènes triphasiques, soit en utilisant le contact triple avec un solide (coalesceurs à impact, grilles diverses, dispositifs internes des séparateurs gravitaires), soit en utilisant un agent facilement séparable (cellules à flottation).

Enfin, il faut parfois agiter le mélange pour accroitre les fréquences de collision et de coalescence. La dimension moyenne des particules augmente et conduit à une séparation plus rapide (barattes).

\section{Réacteurs}

Dans les réacteurs chimiques, on cherche à prévoir et maitriser l'aire interfaciale de contact, qui pour des globules sphériques vaut trois fois l'inverse du diamètre, par unité de volume. Dans certains cas de régimes d'écoulement et de type de réacteurs, il y a compétition entre coalescence et fracturation (colonnes à garnissage, colonnes pulsées); dans d'autres, l'écoulement est transitoire et la coalescence règle en partie l'évolution de l'aire interfaciale volumique (réacteurs à bulle).

Il existe aussi de très nombreux procédés de fractionnement de la phase dispersée dans des systèmes plus ou moins complexes : on peut difficilement les classer mais l'aspect général se regroupe bien sous le sigle "relations entre granularité et turbulence ". Dans ces relations interviennent les paramètres de la turbulence en milieu diphasique, les lois de fracturation de bulles/gouttes sollicitées par la turbulence et les lois de coalescence. Cet ensemble de phénomènes usuellement très liés a besoin d'être étudié dans le détail tant manquent les connaissances fondamentales associées.

Le cas des colonnes à garnissage se rapproche de celui des milieux triphasiques (coalesceurs) ou diphasique en milieu poreux dont on parle plus loin.

Les dépenses d'énergie ne sont pas négligeables dans les réacteurs soit par agitation, soit par différence de pression et on remarque leur sensibilité aux phénomènes d'interface. Il existe presque toujours une variance des dépenses d'énergie avec le temps de coalescence, la granularité qui en résulte, ou une propriété interfaciale active sur la coalescence.

A l'extrême, le moussage fait varier dans de fortes proportions tous les paramètres d'un système d'échange : il doit être évité dans la plupart des situations, mais il est parfois recherché. Les lois de moussage (ou d'antimoussage) sont mal connues et remplacées par des idées intuitives ou empiriques bien que des progrès notables aient été effectués dans ce domaine.

\section{Procédés}

De nombreux organes d'équipements industriels travaillent en régime diphasique en opération normale ou accidentelle. On peut citer :

- pompes, hélices;

- singularités diverses : coudes, tés, dérivations, vannes de tous types, diaphragmes, débitmètres;

- injecteurs, mélangeurs, émulseurs;

- diffuseurs, propulsion diphasique;

- turbines;

- diversions, ressauts hydrauliques.

Dans tous ces dispositifs, l'écoulement varie rapidement et l'évolution de la granularité de la phase dispersée dépend essentiellement du rythme des coalescences.

Dans le cas des singularités, il n'y a d'incidence que sur les pertes de pression. Par contre, sur les systèmes à transfert d'énergie, le taux de coalescence affecte directement le fonctionnement de l'organe (par exemple rotor de pompe, diffuseur) et donc les performances du dispositif.

On peut également chercher à favoriser ou à inhiber la coalescence dans certaines applications. Par exemple, on peut vouloir maintenir une granularité fine pour l'utilisa- 
tion de l'émulsion (suspension) dans un organe qui fonctionnerait mal à une granularité plus élevée. Inversement, on peut vouloir activer la coalescence de façon à faire rapidement grossir le diamètre des globules en vue d'une séparation plus rapide.

Dans certains cas, c'est l'émulsion elle-même qui est l'objet du traitement : on cherche alors à obtenir une distribution de diamètres donnée à l'avance, une stabilité de la granularité fixée (ou l'inverse); là encore, le contrôle de la coalescence est un moyen essentiel du procédé.

\section{Cas triphasiques}

On trouve des cas ou la coalescence en milieu triphasique a une importance industrielle dans les procédés spéciaux principalement mais aussi dans des processus naturels.

Le cas le plus fréquent est celui des milieux granulaire. En voici quelques exemples :

- milieu poreux : gisements de pétrole, récupération assistée;

- milieux "granulaires " : garnissages, grilles de coalescence, impact, colonnes diverses, internes de séparateurs.

On trouve d'autres cas de coalescence en présence d'une paroi dans les séparateurs (plaques), les agitateurs (ailettes).

Quelques cas de milieux triphasiques gaz ou liquideliquide-solide se trouvent dans le transport par conduite et le draguage par air-lift.

Enfin, le cas gaz-liquide-liquide, c'est-à-dire un mélange gaz-émulsion est utilisé en séparation par flottation, rencontré dans des puits de pétrole et les séparateurs primaires, en dépollution marine.

\section{Secteurs d'activités concernés}

Comme d'habitude, on peut classer tout procédé par discipline scientifique et finalité. Nous avons essayé de dresser un tableau des grands secteurs d'activité en indiquant à chaque fois le type de mélange impliqué et les appareils ou procédés mis en cause. La liste des secteurs indiqués est exposée sur le tableau ci-contre.

\section{Principales activités} de recherche connexes

Les progrès scientifiques sont particulièrement intéressants pour l'industrie dans ce domaine du fait d'une carence importante, principalement due à la difficulté d'étude des phénomènes interfaciaux.

Voici les activités de recherche les plus proches du sujet :

- Physique des liquides. Cohésion moléculaire

- Physique des interfaces. Etude de propriétés interfaciales. Mesure des propriétés physiques des interfaces. Tensiométrie Viscosimétrie interfaciale

- Modélisation physique des liquides et des interfaces (théories physiques et mécaniques)
Liste des secteurs indiqués

\begin{tabular}{|c|c|c|}
\hline \multicolumn{2}{|c|}{ SECTEUR } & $\begin{array}{l}\text { AUTRES ACTIVITES } \\
\text { INCLUSES }\end{array}$ \\
\hline Pétrole & $\begin{array}{l}\text { gisements } \\
\text { forage } \\
\text { production } \\
\text { chimie } \\
\text { produits } \\
\text { pétroliers }\end{array}$ & $\begin{array}{l}\text { récupération assistée } \\
\text { boues, laitiers, ciments } \\
\text { process, transport } \\
\text { raffinage, conversion } \\
\text { bitume }\end{array}$ \\
\hline Nucléaire & $\begin{array}{l}\text { ébullition } \\
\text { sécurité }\end{array}$ & $\begin{array}{l}\text { générateurs de vapeur } \\
\text { perte de réfrigérant, } \\
\text { ébullition, fuites }\end{array}$ \\
\hline \multicolumn{2}{|c|}{$\begin{array}{l}\text { Conversion } \\
\text { d'énergie }\end{array}$} & $\begin{array}{l}\text { production classique, énergie } \\
\text { solaire, électrolyse, échan- } \\
\text { geurs à contact direct, pom- } \\
\text { pes à chaleur, réfrigération, } \\
\text { stockage d'énergie calorifique }\end{array}$ \\
\hline Chimie & $\begin{array}{l}\text { réacteurs } \\
\text { fermenteurs } \\
\text { contacteurs } \\
\text { catalyse }\end{array}$ & $\begin{array}{l}\text { fractionnement, distillation, } \\
\text { réacteurs à bulles } \\
\text { chimie fine } \\
\text { extraction liquide-liquide } \\
\text { réacteurs catalytiques tripha- } \\
\text { siques }\end{array}$ \\
\hline \multicolumn{2}{|l|}{ Mousses } & $\begin{array}{l}\text { antimousses, surfactants } \\
\text { mousses industrielles, réac- } \\
\text { teurs à mousse, élastomères } \\
\text { aérés }\end{array}$ \\
\hline \multicolumn{2}{|l|}{ Peintures } & $\begin{array}{l}\text { vernis, produits de protection, } \\
\text { aérosols, émulsifiants }\end{array}$ \\
\hline \multicolumn{2}{|c|}{$\begin{array}{l}\text { Hygiène (Beauté, } \\
\text { Pharmacie, Médecine) }\end{array}$} & $\begin{array}{l}\text { crèmes, pâtes, émulsifiants, } \\
\text { génie biologique }\end{array}$ \\
\hline \multicolumn{2}{|c|}{ Assainissement } & $\begin{array}{l}\text { assainissement urbain, indi- } \\
\text { viduel, dépollution indus- } \\
\text { trielle }\end{array}$ \\
\hline \multicolumn{3}{|c|}{ Dépollution océanique } \\
\hline \multicolumn{2}{|c|}{ Environnement } & $\begin{array}{l}\text { hydrologie, océanologie, } \\
\text { pollutions naturelles et acci- } \\
\text { dentelles }\end{array}$ \\
\hline \multicolumn{2}{|c|}{ Agro-alimentaire } & $\begin{array}{l}\text { produit laitiers, conservation } \\
\text { de produits, émulsions ali- } \\
\text { mentaires, production agri- } \\
\text { cole, engrais, aliments pour } \\
\text { les animaux }\end{array}$ \\
\hline \multicolumn{2}{|c|}{ Génie naval } & $\begin{array}{l}\text { génie océanique, offshore, } \\
\text { propulsion (hélice, jets) } \\
\text { diphasique }\end{array}$ \\
\hline \multicolumn{2}{|l|}{ Dragage } & $\begin{array}{l}\text { remontée de déblais par } \\
\text { gaz-lift } \\
\text { jets cavitants }\end{array}$ \\
\hline \multicolumn{2}{|l|}{ Moteurs } & injection, carburation \\
\hline
\end{tabular}

Nota : seuls les dénominations génériques des secteurs ont été mentionnés dans le tableau. Les autres sont sousentendus. 


\begin{tabular}{|c|c|c|c|c|c|}
\hline \multicolumn{6}{|c|}{ La coalescence dans I'industrie } \\
\hline & Gaz-liquide & Huile-eau & $\begin{array}{c}\text { Autres } \\
\text { liquide-liquide }\end{array}$ & micro-émulsions & colloïdes \\
\hline $\begin{array}{l}\text { Ecoulements en } \\
\text { conduites, } \\
\text { singularités }\end{array}$ & $\begin{array}{l}\text { Pétrole forage, Pétrole } \\
\text { production } \\
\text { Nucléaire ébullition, } \\
\text { Nucléaire sécurité, } \\
\text { Conversion d'énergie }\end{array}$ & \begin{tabular}{|l|} 
Pétroleproduction \\
Conversion \\
d'énergie
\end{tabular} & Agro-alimentaire & Pétrole gisements & Peintures \\
\hline $\begin{array}{l}\text { Pompes } \\
\text { Hélices }\end{array}$ & $\begin{array}{l}\text { Pétrole production } \\
\text { Nucléaire sécurité } \\
\text { Conversion d'énergie }\end{array}$ & \begin{tabular}{|l|} 
Pétroleproduction \\
Conversion \\
d'énergie
\end{tabular} & Agro-alimentaire & & \\
\hline $\begin{array}{l}\text { Injecteurs } \\
\text { Emulseurs hydrauliques }\end{array}$ & $\begin{array}{l}\text { Conversion d'énergie } \\
\text { Assainissement }\end{array}$ & Agro-alimentaire & $\begin{array}{l}\text { Agro-alimentaire } \\
\text { Chimie } \\
\text { contacteurs }\end{array}$ & . & $\begin{array}{l}\text { Peintures } \\
\text { Hygiène }\end{array}$ \\
\hline $\begin{array}{l}\text { Mélangeurs, agitateurs } \\
\text { Emulseurs mécaniques }\end{array}$ & Assainissement & \begin{tabular}{|l|}
$\begin{array}{l}\text { Pétroleproduction } \\
\text { Pétrole chimie }\end{array}$ \\
Agro-alimentaire
\end{tabular} & $\begin{array}{l}\text { Agro-alimentation } \\
\text { Chimie contac- } \\
\text { teurs }\end{array}$ & Agro-alimentaire & $\begin{array}{l}\text { Agro-alimentaire } \\
\text { Peintures } \\
\text { Hygiène }\end{array}$ \\
\hline $\begin{array}{l}\text { Diffuseurs } \\
\text { Propulsion }\end{array}$ & $\begin{array}{l}\text { Conversion d'énergie } \\
\text { Génie naval - Moteurs }\end{array}$ & & & & \\
\hline $\begin{array}{l}\text { Déversoirs, ressauts } \\
\text { Turbines }\end{array}$ & $\begin{array}{l}\text { Conversion d'énergie } \\
\text { Environnement }\end{array}$ & & & & \\
\hline $\begin{array}{c}\text { gravitaires } \\
\text { Séparateurs centrifuges } \\
\text { autres } \\
\end{array}$ & $\begin{array}{l}\text { Pétrole production } \\
\text { Chimie } \\
\text { Chimie }\end{array}$ & \begin{tabular}{|l|} 
Pétroleproduction \\
Conversion \\
d'énergie \\
Chimie \\
\end{tabular} & & & $\begin{array}{l}\text { Agro-alimentaire } \\
\text { Génie biologique } \\
\text { Hygiène }\end{array}$ \\
\hline Réacteurs & $\begin{array}{l}\text { Chimie réacteurs } \\
\text { Chimie fermenteurs }\end{array}$ & $\begin{array}{l}\text { Chimie } \\
\text { contracteurs }\end{array}$ & $\begin{array}{l}\text { Chimie } \\
\text { contracteurs }\end{array}$ & & \\
\hline $\begin{array}{l}\text { Triphasique + } \\
\text { solide }\end{array}$ & $\begin{array}{l}\text { Draguage } \\
\text { Chimie réacteurs } \\
\text { Chimie catalyse } \\
\text { Pétrole gisements } \\
\text { Pétrole chimie }\end{array}$ & $\begin{array}{l}\text { Pétrole gisements } \\
\text { Pétroleproduction }\end{array}$ & & $\begin{array}{l}\text { Pétrole gisements } \\
\text { Pétrole production }\end{array}$ & \\
\hline Triphasique + gaz & & $\begin{array}{l}\text { Dépollution océa- } \\
\text { nique } \\
\text { Pétroleproduction }\end{array}$ & & & \\
\hline $\begin{array}{l}\text { Fabrication de } \\
\text { produits }\end{array}$ & Mousses & \begin{tabular}{|l|} 
Hygiène \\
Produits pétroliers \\
émulsionnés \\
Agro-alimentaire \\
\end{tabular} & $\begin{array}{l}\text { Génie biologique } \\
\text { Hygiène } \\
\text { Agro-alimentaire }\end{array}$ & \begin{tabular}{|l|} 
Produits pétroliers \\
émulsionnés \\
Peintures \\
Hygène
\end{tabular} & $\begin{array}{l}\text { Agro-alimentaire } \\
\text { Génie biologique } \\
\text { Peintures } \\
\text { Hygiène }\end{array}$ \\
\hline $\begin{array}{l}\text { Etude de situations } \\
\text { naturelles }\end{array}$ & $\begin{array}{l}\text { Environnement } \\
\text { Hygiène }\end{array}$ & Pétrole gisements & Génie biologique & & \\
\hline
\end{tabular}

\section{- Adsorption}

- Couches monomoléculaires. Couches minces. Propriétés des couches interfaciales et/ou surfaciques. Surfactants.

- Emulsions. Théorie. Modèles. Métrologie des émulsions (granularité)

- Rhéologie des émulsions, des suspensions, des suspensions colloïdales.

- Aggrégation, flocculation. Effets électriques dans les suspensions et les émulsions.

- Films minces. Attraction moléculaire. Cohésion moléculaire. Stabilité des films minces. Draînage des films minces. Mousses.

- Mécanique des suspensions, des émulsions, des mélanges gaz-liquide.

- Transport di- et triphasique, énergétique di-et triphasique. Pertes de pession. Concentration. Glissement. Débits critiques. Vitesse du "son" dans les mélanges.
- Cavitation, ébullition, condensation.

- Ecoulement diphasiques en milieu granulaire, en milieu poreux.

- Génie chimique, réacteurs gaz-liquide et liquide-liquide. Contacteurs, distillation, garnissages, séparation, moussage. - Coalescence, coalescence globule-interface, 2 globules, en lit dense de globules, en milieu turbulent. Modèles de coalescence. Fréquences de coalescence. Temps de coalescence.

- Fracturation de globules, stabilité des gouttes et des bulles (en écoulement cisaillé, turbulent). Distributions des diamètres.

Cette liste n'est probablement pas exhaustive. On remarquera la grande variété des spécialistes impliquées dans le phénomène de coalescence. Ceci explique sans doute la lenteur des progrès dans le domaine. 


\section{Discussion}

Président : M. J. DUPORT

M. Le Président. - Je remercie beaucoup M. Fitremann. Il nous reste 10 minutes pour la discussion.

M. BONNIN, - Je voudrais une précision à propos de votre cliché $n^{\circ} 3$ : est-ce qu'il s'agit de bulles face à face, de gouttes, ou indifféremment des unes ou des autres?

M. FItREMANN. - J'ai présenté surtout le phénomène "gazliquide ". Il s'agit de deux bulles qui se déforment. Mais le phénomène est général : cela peut s'appliquer à deux gouttes... En particulier le phénomène de molécules absorbées se produit quand il $\mathrm{y}$ a des gouttes en présence.

\section{BonNIN. - C'est le fond de ma question.}

M. FitremanN. - Lorsqu'il y a un gaz et un liquide il ne peut y avoir qu'un surfactant, qui est toujours dans la phase liquide. Mais quand il $\mathrm{y}$ a des phénomènes "liquide-liquide " il y a deux surfactants, l'un dans chaque phase, et c'est par l'effet de cette double action des molécules qu'on stabilise l'interface.

M. Le Président. - Est-ce que vous pensez que le groupe doit s'intéresser à l'ensemble des dispersions, y compris la dispersion dans un milieu continu gazeux avec phase liquide dispersée ?. Vous avez indiqué au début que les problèmes de coalescence s'y présentent de façon différente, en ce sens que chaque fois qu'il y a choc il y a coalescence...

Est-ce que vous avez eu une réflexion entre vous à ce sujet ? Je n'ai pas personnellement d'orientation. Je comprends qu'il y a peut-être davantage de sujets de recherche sur la coalescence dans les cas où la phase continue est liquide... En tout cas si l'on devait s'intéresser dans le groupe à la phase liquide dispersée dans un gaz, il y aurait lieu d'y introduire les problèmes de séparateurs de gouttelettes, et aussi peut-être, les phénomènes naturels, les problèmes atmosphériques.

M. Fitremann. - et aussi les problèmes de condensation.

M. Le Président. - En ce qui concerne le domaine industriel - les "secteurs", comme vous les appelez - vous avez très justement dit que votre recensement ne prétendait pas être exhaustif; je crois qu'il faut accorder une certaine importance à la minéralurgie qui met en œuvre des systèmes triphasiques, des systèmes de flottation qui sont justiciables certainement de progrès.

M. FitremanN. - Vous avez demandé si nous pensions que le Groupe devait s'intéresser à l'ensemble des dispersions. Pour le moment nous n'avons pas fait de distinction; nous nous sommes limités au cas "liquide-liquide ".

La coalescence du milieu " gaz/liquide " est très rapide, ce qui fait que les phénomènes sont très difficiles à analyser.

Dans le cas "liquide/liquide", le phénomène est de l'ordre de 5 secondes, et les chercheurs ont pu analyser le problème. Maintenant ils pourraient peut-être transposer leurs connaissances au phénomène " gaz/liquide ", en tenant compte du fait qu'il faut aller beaucoup plus vite.

On n'exclut rien; mais on ne pense pas beaucoup au phénomène de coalescence de deux gouttes qui est toujours très rapide; mais c'est un phénomène qui ne sera pas oublié, si quelqu'un s'y intéresse.

M. Le Président. - Vous avez également signalé les applications des connaissances en matière de coalescence dans le domaine du génie biologique. On peut se poser la question de l'application de ces connaissances dans le domaine à proprement parler de la biologie... et notamment de la biologie humaine. Il pourrait être intéressant d'avoir un contact avec des biologistes et des médecins.

M. Fitremann. - Il y a un groupe à l'Institut Pasteur qui s'occupe d'application de la physico-chimie des interfaces à la biologie... Nous connaissons ces messieurs; nous ne les avons pas encore rencontrés, mais on le fera pour savoir ce qu'ils pensent de ces études pour des applications purement biologiques.

M. J. BERNARD. - On a parlé de triphasique tout à l'heure. Je suis intéressé par le triphasique puisqu'il s'agit, dans le cadre du G.I.P., de pomper des nodules... C'est pourquoi je voudrais poser une question : Est-ce que la coalescence est plutôt favorisée ou plutôt défavorisée par la présence de particules solides, par exemple, dans un milieu "eau/air" ?

M. FitremanN. - Sur le plan "mécanique des fluides" la présence de particules est un peu défavorisante... Les particules vont augmenter le nombre des collisions. Par contre les impuretés contenues dans le solide peuvent jouer un rôle de surfactant et par conséquent favoriser la coalescence sur le plan «moléculaire ". Par conséquent c'est un problème à étudier plus dans le détail.

M. LECOFFRE. - Est-ce qu'il existe des micro-émulsions ?

M. FITREMANN. - Oui. J'ai dit que ce sont des états thermodynamiquement stables. Ils se comportent comme une phase à elle toute seule.

Si vous avez un mélange "eau/huile ", et que vous mettez ce qu'il faut pour fabriquer des micro-émulsions, vous pouvez obtenir le résultat cherché. Vous avez donc trois phases en présence. Vous pouvez avoir deux micro-émulsions, et tout cela est quand même en équilibre thermodynamique, par conséquent stable...

M. LEFEBVRE. - Quels sont les pourcentages de bulles d'air dans l'eau ?... Et comment faire pour éviter les phénomènes de coalescence parce qu'on veut éviter l'opacité...

S'agissant de faire des écrans blancs, des vitres blanches, le taux de gaz qui intervient dans la coalescence est un point important. Pour l'eau et l'air, ce taux critique varie beaucoup : pour de l'eau très purifiée c'est $5 \%$; pour de l'eau du robinet c'est $50 \%$; et l'on peut mélanger jusqu'à $95 \%$ en mettant des additifs. ment.

M. BONNIN. - En plus, cela dépend des conditions d'écoule-

M. CAZENAVE. - Je veux maintenant remercier les conférenciers pour nous avoir fait passer un maximum d'informations de la façon la plus brillante. On apprécie beaucoup leur travail à la Société hydrotechnique de France; faute de temps, on a un peu coupé la fin de l'exposé de M. GIMENEZ. Il serait intéressant qu'il nous indique par une petite note les grandes lignes de son programme et que nous puissions, s'il en est d'accord, en faire état dans le compte-rendu de cette demi-journée.

M. Le Président. - Je tiens à m'associer aux compliments et félicitations que M. CAZENAVE a adressés à M. Gimenez. Je tiens aussi à remercier vivement $\mathrm{M}$. FITREMANN pour son exposé et pour l'excellent travail qu'il accomplit à la tête du groupe coalescence. Pour ma part j'ai l'impression d'avoir été un président un peu sévère sur l'horaire : j'ai choisi le parti de la rigueur.

Je vous remercie de votre attention. 


\section{Le groupe de réflexion}

\section{Coalescence}

Créé par décision du Comité technique de la Société hydrotechnique de France le 13 mai 1982, le groupe de travail " Coalescence " animé par Jean-Michel Fitremann, réunit actuellement une quarantaine de personnes, industriels et chercheurs par moitié environ.

\section{$\therefore$}

\section{Objectifs}

Faciliter le contact et les échanges d'informations entre chercheurs et entre chercheurs et industriels :

- informer les participants d'activités les intéressant, de recherches ayant lieu sur le sujet en France et à l'étranger, des préoccupations de l'industrie etc.,

- organiser tous séminaires, voyages d'études, visites et enquêtes utiles aux participants:

- proposer ou suggérer :

- des thèmes de recherche

- des actions de coopération, actions concertées, actions de financement

auprès d'autorités compétentes à la suite des études prospectives; - participer à des actions existantes oủ des informations sur ce thème seraient bienvenues (comités techniques, scientifiques, etc.).

\section{Vie du groupe}

En principe, une réunion plénière annuelle permet d'exposer à tous les résultats du travail de petites cellules plus faciles à actionner.

Dès à présent, deux "bureaux " se sont réunis à des fins d'enquête :

1. Le bureau "recherche" qui a effectué une enquête en France auprès des laboratoires afin de mieux connaitre l'existant et le manquant sur le sujet, les effectifs et les moyens dont nous disposons. Les résultats de cette enquête ont fait l'objet d'un rapport.

On effectuera par la suite une série de visites de laboratoires associées à des séminaires au rythme de deux jours deux fois par an, organisés par le bureau "recherche". La participation à ces réunions est ouverte à tous les intéressés.

2 . Le bureau "industrie " réuni récemment, procède actuellement à une enquête sur les besoins du secteur économique en connaissances fondamentales sur ce sujet. Le rapport est destiné à être diffusé auprès des laboratoires et des diverses directions de la recherche.

\section{Documents}

Les documents et informations concernant la vie du groupe sont disponibles pour toute personne intéressée en s'adressant à la Société Hydrotechnique de France ou à Jean-Michel Fitremann (Hydroscience, 10, chemin du Bout des Landes, 44300 Nantes. Tél. (40) 76.72 .80$)$ 
Theorie des Fleuves Flanche 1 inI.

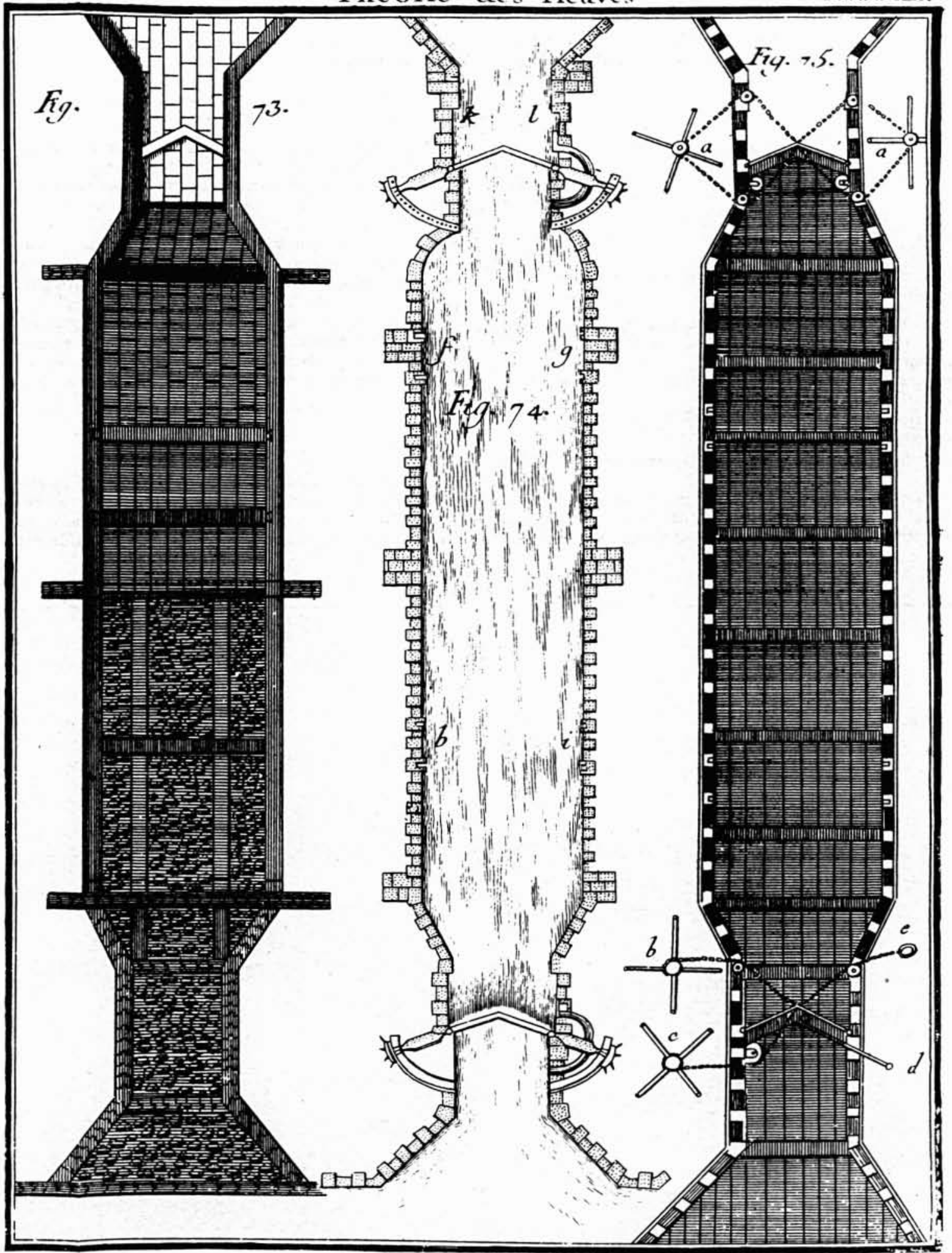

